\title{
New 2D-DOA and Angular Spread Estimation method for Coherently Distributed Sources
}

\author{
Hui Yao, Ying Wu \\ Zhenzhou Information Science and Technology Institute, Zhenzhou, 450002, China \\ E-mail: hui56_y@126.com
}

\begin{abstract}
In many two-dimensional Direction of Arrival estimation approaches for Coherently distributed source, the computational complexity induced by high-dimensional peakfinding searching is normally highly demanding. We propose a low-complexity estimation algorithm by using the approximation to the steering to vector for small angular extensions, which estimates the nominal DOA using the phase information of the estimated invariance structured diagonal matrix. Then angular spread can be estimated form the module of the steering responses with the angular weighting function of CD sources. The proposed algorithm provides a good estimation performance while requiring low complexity and it can be use any array without restrictions. In addition, this algorithm can obtain the nominal DOA without the priori knowledge of the distribution type. The efficiency of the algorithm is validated by computer simulations.
\end{abstract}

Keywords-Coherently distributed source; DOA; Angular spread; Cumulants

\section{INTRODUCTION}

Many classic direction of arrival (DOA) estimation algorithms assume impinging sources to be point sources during the field of array signal processing. However, in applications such as radar and mobile communications, signal energy spreads to an unknown angular range because of scattering, and then arrives at receivers within a continuous angular range. This space propagation characteristic of signals strongly affects the above assumption, which results in performance deterioration of classic algorithms such as MUSIC and ESPRIT. In order to solve this problem, parameterized distributed sources model ${ }^{[1-3]}$ is usually employed in DOA estimation.

The authors of [1] extend the perspective of signal subspace and noise subspace into distributed sources model, and make further efforts to propose a MUSIC-like parameter estimation method called DSPE algorithm. This method calls for a 2-D peak searching and therefore brings about heavy computation. On the basis of DSPE algorithm, [4] extends ESPRIT into distributed sources model and then obtains the rotational invariance matrix by Taylor approximation, which avoids 2-D peak searching while special array structure is needed. Generalized Capon beamspace forming method is used to estimate parameters of distributed sources in [5], and it still needs a 2-D searching although computation cost is reduced. [6] introduces a covariance fitting method, which dose not need high dimension searching and has low complexity, but it need the original information of sources' DOA.
For the two-dimension parameters estimation for distributed sources, a low-complexity estimation algorithm has been proposed in [7] in which bases on the eigenstructure between the steering matrix and signal subspace, it converts a 2-D searching problem to a 1-D problem. However, it uses a pair of uniform circular arrays which increases the difficulty of hardware realization. Another approach is proposed in [8], where the 2D-DOA and angular spread of the sources is estimated by using the LS-VESPA algorithm based on the newly designed array geometry. In this paper, we estimate the nominal DOA using the phase information of the estimated invariance structured diagonal matrix, which constructed form the fourth-order cumulate-based technique. Then angular spread can be estimated form the module of the steering responses with the angular weighting function of $\mathrm{CD}$ sources. In essence, the contributed of this paper is the application of the combination of distributed signal model, cumulant-based technique, and ESPRIT as a collective study.

\section{DISTRIBUTED SOURCE MODEL}

Assume that stationary signals impinge on an uniform circular array of $M$ sensors from $q$ distributed narrowband far-field sources. The output of the array can be expressed as

$$
\boldsymbol{X}(t)=\sum_{i=1}^{q} \iint \boldsymbol{a}(\theta, \varphi) s_{i}(t) g_{i}\left(\theta, \varphi ; \boldsymbol{\mu}_{i}\right) d \theta d \varphi+\boldsymbol{n}(t)
$$

Where the steering vector of the array $\boldsymbol{a}(\theta, \varphi)=\left[e^{j \Delta \cos \varphi \cos (\theta)}, e^{j \Delta \cos \varphi \cos (\theta-2 \pi / M)}, e^{j \Delta \cos \varphi \cos (\theta-2 \pi(M-1) / M)}\right]^{\mathrm{T}}$, $\Delta=2 \pi r / \lambda$ with $r$ the radius of the UCA and $\lambda$ the wavelength of the arriving wave, $s_{i}(t)$ is complex envelop of the signal, $g_{i}\left(\theta, \varphi ; \boldsymbol{\mu}_{i}\right)$ is a deterministic angular weighting function, and is parameterized by the vector $\boldsymbol{\mu}=\left(\theta_{0}, \sigma_{\theta}, \varphi_{0}, \sigma_{\varphi}\right)$ denoting the nominal azimuth DOA $\theta_{0}$, angular spread $\sigma_{\theta}$ of the azimuth DOA, the nominal elevation DOA $\varphi_{0}$, and angular spread of the elevation DOA.

$\boldsymbol{b}(\boldsymbol{\mu})$ is the generalized steering vector defined as

$$
\boldsymbol{b}(\boldsymbol{\mu})=\iint \boldsymbol{a}(\theta, \phi) g(\theta, \varphi ; \boldsymbol{\mu}) d \theta d \varphi
$$

The coherently distributed source model (1) can be expressed as

$$
\boldsymbol{X}(t)=\sum_{i=1}^{q} s_{i}(t) \boldsymbol{b}\left(\boldsymbol{\mu}_{i}\right)+\boldsymbol{n}(t)
$$


Assume that the deterrministic angular weighting function $g(\theta, \varphi ; \boldsymbol{\mu})$ has the Gaussian shape

$$
g(\theta, \varphi ; \boldsymbol{\mu})=\frac{1}{2 \pi \sigma_{\theta} \sigma_{\varphi}} e^{-1 / 2\left(\left(\theta-\theta_{0}\right)^{2} / \sigma_{\theta}^{2}+\left(\varphi-\varphi_{0}\right)^{2} / \sigma_{\varphi}^{2}\right)}
$$

Then, the closed form of the generalized steering vector can be written as

$$
\begin{aligned}
& {[\boldsymbol{b}(\boldsymbol{\mu})]_{m}=\iint[\boldsymbol{a}(\theta, \phi)]_{k} g(\theta, \boldsymbol{\varphi} ; \boldsymbol{\mu}) d \theta d \varphi} \\
& \approx e^{j \Delta \cos \varphi_{0} \cos \left(\theta_{0}-\gamma_{m}\right)} \iint e^{-j \Delta\left(\tilde{\varphi} \sin \varphi_{0} \cos \left(\theta_{0}-\gamma_{m}\right)+\tilde{\theta} \cos \varphi_{0} \sin \left(\theta_{0}-\gamma_{m}\right)\right)} \\
& \times g\left(\tilde{\theta}+\theta_{0}, \tilde{\varphi}+\varphi_{0} ; \boldsymbol{\mu}\right) d \tilde{\theta} d \tilde{\varphi} \\
& =e^{j \Delta \cos \varphi_{0} \cos \left(\theta_{0}-\gamma_{m}\right)}[\boldsymbol{G}(\boldsymbol{\mu})]_{m}
\end{aligned}
$$

Where []$_{m}$ indicates the $m$ th element of a vector and $[\boldsymbol{G}(\boldsymbol{\mu})]_{m}=e^{-1 / 2\left(\sigma_{\theta}^{2} \sin ^{2} \varphi_{0} \cos ^{2}\left(\theta-\gamma_{m}\right)+\sigma_{\varphi}^{2} \cos ^{2} \varphi_{0} \sin ^{2}\left(\theta-\gamma_{m}\right)\right)}$ for small angular spread $\sigma_{\theta}$ and $\sigma_{\varphi}$.

\section{PARAMETER ESTIMATION}

Assume that the received signals at $m$ th sensor is given as follows

$$
x_{m}(t)=\sum_{i=1}^{q} b_{m}\left(\mu_{i}\right) s_{i}(t)+n_{m}(t)
$$

The cumulant matrix $\boldsymbol{C}_{11}$ is defined as

$$
\begin{aligned}
& \boldsymbol{C}_{11}=\operatorname{cum}\left(x_{1}(t), \boldsymbol{X}(t), x_{1}^{*}(t), \boldsymbol{X}^{*}(t)\right) \\
& =\operatorname{cum}\left(\sum_{i=1}^{q} b_{1}\left(\mu_{i}\right) s_{i}(t), \sum_{k=1}^{q} \boldsymbol{b}_{k}\left(\mu_{k}\right) s_{k}(t),\right. \\
& \left.\sum_{j=1}^{q} b_{1}^{*}\left(\mu_{j}\right) s_{j}(t), \sum_{l=1}^{q} \boldsymbol{b}_{l}^{*}\left(\mu_{l}\right) s_{l}(t)\right) \\
& +\operatorname{cum}\left(n_{1}(t), \boldsymbol{n}(t), n_{1}^{*}(t), \boldsymbol{n}(t)\right) \\
& =\sum_{i=1}^{q} \sum_{j=1}^{q} \sum_{k=1}^{q} \sum_{l=1}^{q} b_{1}\left(\mu_{i}\right) b_{1}^{*}\left(\mu_{j}\right) \boldsymbol{b}_{k} \boldsymbol{b}_{l}^{*} \\
& \times \operatorname{cum}\left(s_{i}(t), s_{j}^{*}(t), s_{k}(t), s_{l}^{*}(t)\right) \\
& =\sum_{i=1}^{q}\left|b_{1}\left(\mu_{i}\right)\right|^{2} \boldsymbol{b}_{i} \boldsymbol{b}_{i}^{\mathrm{H}} \gamma_{4 s_{i}}=\boldsymbol{B} \boldsymbol{\Lambda} \boldsymbol{B}^{\mathrm{H}}
\end{aligned}
$$

Where $\gamma_{4 s_{i}}$ is the fourth-order cumulants of the source signals,

$$
\boldsymbol{\Lambda}=\operatorname{diag}\left\{\gamma_{4 s_{1}}\left|b_{1}\left(\mu_{1}\right)\right|^{2}, \gamma_{4 s_{2}}\left|b_{1}\left(\mu_{2}\right)\right|^{2}, \cdots, \gamma_{4 s_{q}}\left|b_{1}\left(\mu_{q}\right)\right|^{2}\right\} \text {. }
$$

Similarly, $\boldsymbol{C}_{12}$ and $\boldsymbol{C}_{13}$ are defined as

$$
\begin{aligned}
& \boldsymbol{C}_{12}=\operatorname{cum}\left(x_{1}(t), \boldsymbol{X}(t), x_{2}^{*}(t), \boldsymbol{X}^{*}(t)\right)=\boldsymbol{B} \boldsymbol{\Phi}_{1} \boldsymbol{\Lambda} \boldsymbol{B}^{\mathrm{H}} \\
& \boldsymbol{C}_{13}=\operatorname{cum}\left(x_{1}(t), \boldsymbol{X}(t), x_{2}^{*}(t), \boldsymbol{X}^{*}(t)\right)=\boldsymbol{B} \boldsymbol{\Phi}_{2} \boldsymbol{\Lambda} \boldsymbol{B}^{\mathrm{H}}
\end{aligned}
$$

Where $\boldsymbol{\Phi}_{1}=\operatorname{diag}\left\{\frac{b_{2}\left(\mu_{1}\right)}{b_{1}\left(\mu_{1}\right)}, \frac{b_{2}\left(\mu_{2}\right)}{b_{1}\left(\mu_{2}\right)}, \cdots, \frac{b_{2}\left(\mu_{q}\right)}{b_{1}\left(\mu_{q}\right)}\right\}$,

$$
\boldsymbol{\Phi}_{2}=\operatorname{diag}\left\{\frac{b_{3}\left(\mu_{1}\right)}{b_{1}\left(\mu_{1}\right)}, \frac{b_{3}\left(\mu_{2}\right)}{b_{1}\left(\mu_{2}\right)}, \cdots, \frac{b_{3}\left(\mu_{q}\right)}{b_{1}\left(\mu_{q}\right)}\right\} \text {. }
$$

The generalized steering vector $\boldsymbol{B}$ is assumed to be full rank, $\boldsymbol{\Lambda}$ is also full rank. Thus the ESPRIT algorithm could be applied to estimate $\boldsymbol{\Phi}_{1}$ and $\boldsymbol{\Phi}_{2}$. The total cumulant matrix $\boldsymbol{C}$ can be written as

$$
\boldsymbol{C}=\left[\begin{array}{l}
\boldsymbol{C}_{11} \\
\boldsymbol{C}_{12} \\
\boldsymbol{C}_{13}
\end{array}\right]=\left[\begin{array}{l}
\boldsymbol{B} \\
\boldsymbol{B} \Phi_{1} \\
\boldsymbol{B} \Phi_{2}
\end{array}\right] \boldsymbol{A B}=\boldsymbol{B}_{1} \boldsymbol{\Lambda} \boldsymbol{B}
$$

By performing an singular value decomposition of $\boldsymbol{C}$, we get

$$
\boldsymbol{C}=\boldsymbol{U} \boldsymbol{\Sigma} \boldsymbol{V}^{\mathrm{H}}\left[\begin{array}{ll}
\boldsymbol{U}_{s} & \boldsymbol{U}_{N}
\end{array}\right]\left[\begin{array}{cc}
\boldsymbol{\Sigma}_{s} & 0 \\
0 & 0
\end{array}\right] \boldsymbol{V}^{\mathrm{H}}
$$

Consequently, there exists a full rank matrix $\boldsymbol{T}$, such that

$$
\boldsymbol{U}_{s}=\left[\begin{array}{l}
\boldsymbol{U}_{s 1} \\
\boldsymbol{U}_{s 2} \\
\boldsymbol{U}_{s 3}
\end{array}\right]=\left[\begin{array}{l}
\boldsymbol{B} \\
\boldsymbol{B} \Phi_{1} \\
\boldsymbol{B} \boldsymbol{\Phi}_{2}
\end{array}\right] \boldsymbol{T}
$$

The $\boldsymbol{\Phi}_{1}$ and $\boldsymbol{\Phi}_{2}$ can be estimated by the algorithm in [9]. Assume that the $\boldsymbol{C}_{11}, \boldsymbol{C}_{12}$ and $\boldsymbol{C}_{13}$ are the cumulant matrix of the first 3 sensors of the UCA, we would estimate the elements in matrices $\boldsymbol{\Phi}_{1}$ and $\boldsymbol{\Phi}_{2}$ for Gaussian shaped CD sources as follows

$$
\begin{aligned}
& \lambda_{1}^{i}=\frac{b_{2}\left(\mu_{1}\right)}{b_{1}\left(\mu_{1}\right)}=e^{j \Delta \cos \varphi_{i}\left(\cos \left(\theta_{i}-2 \pi / M\right)-\cos \theta_{i}\right)} \\
& \times e^{-\Delta^{2} / 2\left(\sigma_{\theta}^{2} \sin ^{2} \varphi_{i}\left(\cos ^{2}\left(\theta_{i}-2 \pi / M\right)-\cos ^{2} \theta_{i}\right)\right)} \\
& \times e^{-\Delta^{2} / 2\left(\sigma_{\varphi}^{2} \cos ^{2} \varphi_{i}\left(\sin ^{2}\left(\theta_{i}-2 \pi / M\right)-\sin ^{2} \theta_{i}\right)\right)} \\
& \lambda_{2}^{i}=\frac{b_{3}\left(\mu_{1}\right)}{b_{1}\left(\mu_{1}\right)}=e^{j \Delta \cos \varphi_{i}\left(\cos \left(\theta_{i}-4 \pi / M\right)-\cos \theta_{i}\right)} \\
& \times e^{-\Delta^{2} / 2\left(\sigma_{\theta}^{2} \sin ^{2} \varphi_{i}\left(\cos ^{2}\left(\theta_{i}-4 \pi / M\right)-\cos ^{2}\left(\theta_{i}-2 \pi / M\right)\right)\right)} \\
& \times e^{-\Delta^{2} / 2\left(\sigma_{\varphi}^{2} \cos ^{2} \varphi_{i}\left(\sin ^{2}\left(\theta_{i}-4 \pi / M\right)-\sin ^{2} \theta_{i}\right)\right)}
\end{aligned}
$$

Where $\lambda_{1}^{i}$ and $\lambda_{2}^{i}$ are $i$ th element of the matrices $\boldsymbol{\Phi}_{1}$ and $\boldsymbol{\Phi}_{2}$.

From (13) and (14), we can see that the phase information of the matrices $\boldsymbol{\Phi}_{1}$ and $\boldsymbol{\Phi}_{2}$ only contain the nominal DOA, thus we have 
$\hat{\theta}_{i}=$

$$
\begin{gathered}
\operatorname{atan}\left(\frac{\left(\cos \left(\frac{4 \pi}{M}\right)-1\right)-\arg \left(\lambda_{2}^{i} / \lambda_{1}^{i}\right)\left(\cos \left(\frac{2 \pi}{M}\right)-1\right)}{\arg \left(\lambda_{2}^{i} / \lambda_{1}^{i}\right) \sin \left(\frac{2 \pi}{M}\right)-\sin \left(\frac{4 \pi}{M}\right)}\right) \\
\hat{\varphi}_{i}=\operatorname{acos}\left(\frac{\arg \left(\lambda_{1}^{i}\right)}{\Delta\left(\cos \left(\hat{\theta}_{i}-2 \pi / M\right)-\cos \hat{\theta}_{i}\right)}\right)
\end{gathered}
$$

Substituting (15) and (16) into (13) and (14), and after some manipulation, we can obtain the angular spreads of Gaussian shaped angular weighting function of CD sources as follows

$$
\begin{aligned}
& \hat{\sigma}_{\theta}=\sqrt{\frac{\left(b_{2} \ln \left|\lambda_{1}^{i}\right|-b_{1} \ln \left|\lambda_{2}^{i}\right|\right)}{-\Delta^{2} / 2 \sin ^{2} \hat{\varphi}_{i}\left(a_{1} b_{2}-a_{2} b_{1}\right)}} \\
& \hat{\sigma}_{\varphi}=\sqrt{\frac{\left(a_{2} \ln \left|\lambda_{1}^{i}\right|-a_{1} \ln \left|\lambda_{2}^{i}\right|\right)}{-\Delta^{2} / 2 \cos ^{2} \hat{\varphi}_{i}\left(a_{2} b_{1}-a_{1} b_{2}\right)}}
\end{aligned}
$$

Where

$$
\begin{aligned}
& a_{1}=\cos ^{2}\left(\hat{\theta}_{i}-2 \pi / M\right)-\cos ^{2} \hat{\theta}_{i} \\
& a_{2}=\cos ^{2}\left(\hat{\theta}_{i}-4 \pi / M\right)-\cos ^{2} \hat{\theta}_{i} \\
& b_{1}=\sin ^{2}\left(\hat{\theta}_{i}-2 \pi / M\right)-\sin ^{2} \hat{\theta}_{i} \\
& b_{2}=\sin ^{2}\left(\hat{\theta}_{i}-4 \pi / M\right)-\sin ^{2} \hat{\theta}_{i} .
\end{aligned}
$$

Up to now, the parameters of the distributed source have been obtained. Note that the proposed algorithm obtains the nominal azimuth DOA, elevation DOA without the knowledge of the distribution of the angular weighting function. In addition, the proposed algorithm can be applied to also a scenario in which distributed sources may have different angular weighting functions.

\section{SIMULATION EXAMPLES}

In this section, we investigate the performance of the proposed algorithm through some simulation experiments. Assume that the UCA has $M=9$ sensors, the radius is $r=1.5 \lambda$. The two distributed sources have Gaussian shaped angular weighting functions both in azimuth and elevation with parameters $\mu_{1}=\left(-30^{\circ}, 5^{\circ}, 30^{\circ}, 5^{\circ}\right)$ and $\boldsymbol{\mu}_{2}=\left(30^{\circ}, 5^{\circ}, 45^{\circ}, 5^{\circ}\right)$. In the first example, we illustrate the performance of the nominal DOA and angular spread estimators versus SNR. The results are shown in Fig.1, snapshot is 2000 , SNR ranges from $-10 \mathrm{~dB}$ to $30 \mathrm{~dB}$, and 200 Monte Carlo simulations were run to estimate the RMSE of proposed estimates. Where RSME is defined as $R M S E=\sqrt{\left(\tilde{\theta}_{1}-\theta_{1}\right)^{2}+\left(\tilde{\theta}_{2}-\theta_{2}\right)^{2}}$.

According to Fig.1 , the RMSE of nominal DOA and angular spreads estimated by the proposed algorithm are worse than that of DSPE, especially at low SNR. Note that the proposed algorithm provides a sufficiently good estimation accuracy as well as computational simplicity for estimating the nominal DOA and angular spreads without any search, and the computational requirement of DSPE is in general 4-dimensional search.

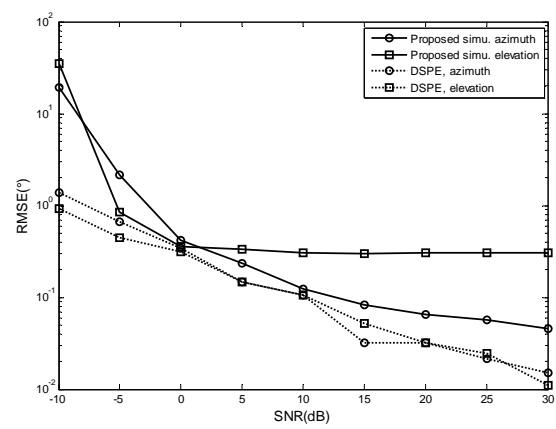

(a) nominal azimuth and nominal elevation

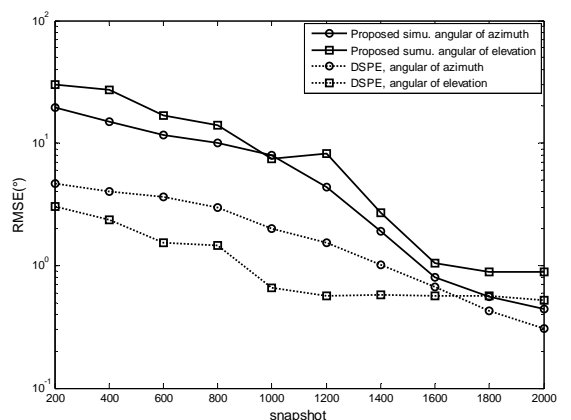

(b) angular spread of azimuth and elevation

Fig.1 The RMSE of nominal DOA and angular spreads versus SNR

In the second example, we numerically illustrate the performance of the nominal DOA and angular spread estimators as a function of number of snapshots, SNR is 20dB, snapshot ranges from 200 to 2000 。

Fig.2 show that the performance of the proposed algorithm is worse than that of DSPE but it is asymptotically efficient when number of snapshots increases, which is due to the properties of cumulant. The RSME of the nominal DOA and angular spread are the same as DSPE when have enough snapshots.

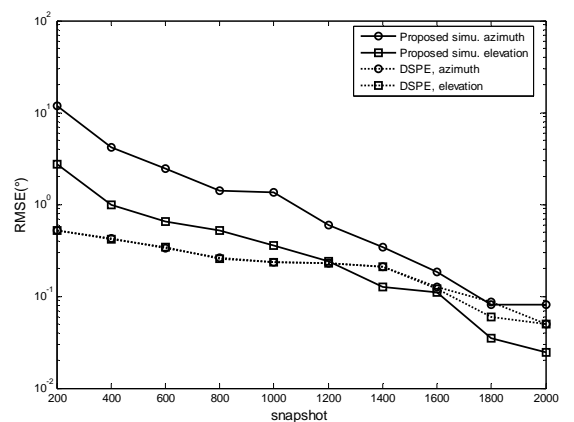

(a) nominal azimuth and nominal elevation 


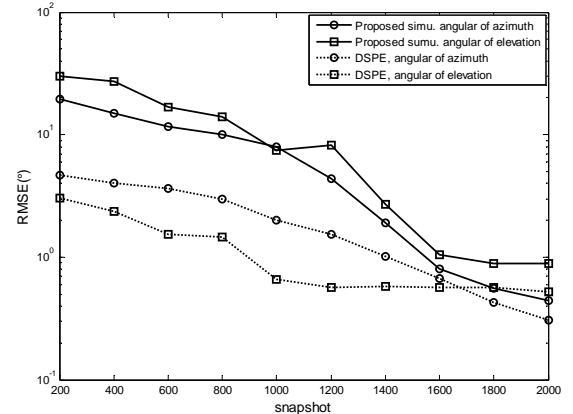

(b) angular spread of azimuth and elevation

Fig.2 The RMSE of nominal DOA and angular spreads versus snapshot

In the third example, we examine the estimation of the RMSE of nominal azimuth and elevation when the angular spread of azimuth and elevation varies without the priori knowledge of the distribution type. Fig.3 show that the nominal DOA estimation performance reduces when the angular extension increases, which introduces the proposed algorithm is strongly influenced by angular spread. However, the proposed algorithm has acceptable performance when the angular spread is modest.

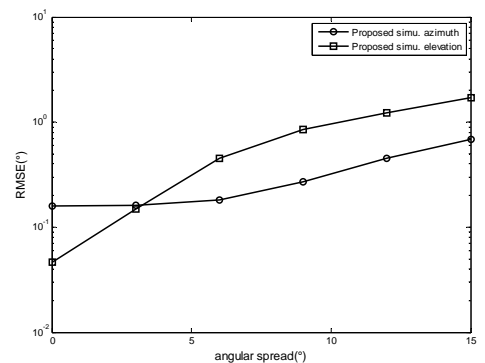

Fig.3 The RMSE of nominal DOA versus angular spread

\section{CONCLUSIONS}

In this paper, we have considered the estimation of the two-dimensional nominal DOA and angular spread of multiple coherently distributed sources. Based on the properties of cumulant, we have proposed a low-complexity algorithm by using the approximation to the steering to vector for small angular extensions, which estimates the nominal DOA using the phase information of the estimated invariance structured diagonal matrix. Then angular spread can be estimated form the module of the steering responses with the angular weighting function of CD sources. The proposed algorithm provides as good an estimation performance as that of DPSE at high SNR and large snapshot while requiring low complexity. In addition, this algorithm can obtain the nominal DOA without the priori knowledge of the distribution type.

\section{REFERENCES}

[1] S. Valaee, B. Champagne, and P. Kabal, "Parametric localization of distributed sources[,” IEEE Transanctions on Signal Processing, vol. 43, No. 9, pp. 2144-2153, 1995.

[2] Z. Zheng, G. J. Li, "Decoupled estimation if the central azimuth and elevation for an incoherently distributed source”, Energy Procedia, vol. 13, pp. 4680-4687, 2011.

[3] Y. H. Han, J. K. Wang, Q. Zhao, et al, “An efficient DOA tracking algorithm for coherently distributed source,” ISCIT, pp. 493-496, 2011.

[4] A.Zoubir, Y. Wang, P. Charge, "The generalized beamforming techniques for estimating the coherently distributed sources," Proc. $8^{\text {th }}$ European Conf. Wireless Technology, pp. 157-160, 2005.

[5] S. Shahbazpanahi, S. Valaee, and M. H. Bastani, "Distributed source localization using ESPRIT algorithm,” IEEE Transactions on Signal Processing, vol. 49, No. 10, pp. 2169-2178, 2001.

[6] A.Zoubir, Y. Wang, P. Charge, "Efficient subspace-based estimator for localization of multiple incoherently distributed sources," IEEE Transactions on Signal Processing, vol. 56, No. 2, pp.532-542, 2009.

[7] J. Lee, I. Song, H. Kwon, and et al, "Low-complexity estimation of 2D DOA for coherently distributed sources,” Signal Processing, vol. 83, pp. 1792-1802, 2003.

[8] G. Y. Zhang, B. Tang, "Estimation of 2D-DOAs and angular spreads for coherently distributed sources using cumulants,",

[9] D. Wang, Y. Wu, "2-D ESPRIT algorithm research based on uniform circular array,” Journal on Communications, vol.27, No.9, pp.89-101, 2006. 